\title{
The advantages of using traditional Chinese medicine as an adjunctive therapy in the whole course of cancer treatment instead of only terminal stage of cancer
}

\author{
Fanghua $\mathrm{Qi}^{1}$, Lin Zhao ${ }^{1}$, Aiyan Zhou ${ }^{1}$, Bo Zhang ${ }^{2}$, Anyuan $\mathrm{Li}^{1}$, Zhixue Wang ${ }^{1, *}$, Junqing Han ${ }^{3, *}$ \\ ${ }^{1}$ Department of Traditional Chinese Medicine, Shandong Provincial Hospital affiliated to Shandong University, Ji'nan, China; \\ ${ }^{2}$ Department of Traditional Chinese Medicine and Traumatology, the Affiliated Hospital of Shandong Academy of Medical Sciences, \\ Ji'nan, Shandong, China; \\ ${ }^{3}$ Department of Tumor Research and Therapy Center, Shandong Provincial Hospital affiliated to Shandong University, Ji'nan, \\ China.
}

Summary Recent studies indicate that Traditional Chinese medicine (TCM) can play an important role in the whole course of cancer treatment such as recovery stages of post-operative, radiotherapy or chemotherapy stages instead of only terminal stage of cancer. In this review, we have summarized current evidence for using TCM as adjuvant cancer treatment in different stages of cancer lesions. Some TCMs (e.g., TJ-41, Liu-jun-zi-tang, PHY906, Coumarin, and Aescine) are capable of improving the post-operative symptoms such as fatigue, pain, appetite, diarrhea, nausea, vomiting, and lymphedema. Some TCMs (e.g., Ginseng, Huang-Qi, BanZhiLian, TJ-48, Huachansu injection, Shenqi fuzheng injection, and Kanglaite injection) in combination with chemo- or radio-therapy are capable of enhancing the efficacy of and diminishing the side effects and complications caused by chemo- and radiotherapy. Taken together, they have great advantages in terms of suppressing tumor progression, relieving surgery complications, increasing the sensitivity of chemo- and radiotherapeutics, improving an organism's immune system function, and lessening the damage caused by surgery, chemo- or radio-therapeutics. They have significant effects on relieving breast cancer-related lymphedema, reducing cancer-related fatigue and pain, improving radiation pneumonitis and gastrointestinal side effects, protecting liver function, and even ameliorating bone marrow suppression. This review of those medicines should contribute to an understanding of Chinese herbal medicines as an adjunctive therapy in the whole course of cancer treatment instead of only terminal stage of cancer, by providing useful information for development of more effective anti-cancer drugs and making more patients "survival with cancer" for a long time.

Keywords: Traditional Chinese medicine (TCM), adjunctive therapy, the whole course of cancer treatment, post-operation, chemotherapy, radiotherapy

\section{Introduction}

Cancer is redefined as a chronic health problem like hypertension and diabetes by the World Health Organization (WHO) and is increasing fast in

\footnotetext{
*Address correspondence to:

Dr. Zhixue Wang, Department of Traditional Chinese Medicine, Dr. Junqing Han, Department of Tumor Research and Therapy Center, Shandong Provincial Hospital affiliated to Shandong University, Ji'nan, China;

E-mail: 756795840@qq.com (Dr. Wang ZX)

E-mail: hanjunqing1960@126.com (Dr. Han JQ)
}

incidence in all regions of the world. It is predicted to be a worldwide important cause of morbidity and mortality in the next few decades. By 2020 in the world approximately 24.6 million people will live with cancer with about $12.5 \%$ of all deaths attributable to cancer, and in China approximately 3.12 million per year or 8,550 new cancer cases per day will emerge with a death toll of 2.5 million; furthermore, the chance of suffering from a malignant tumor is about $22 \%$ for a person in his life $(1,2)$. Thus, in the coming years it is still a huge challenge for cancer prevention and therapy in the world especially in low and middle-income countries such as China. 
Surgery, chemotherapy, radiotherapy, targeted therapy and immunotherapy are examples of anticancer therapies currently being utilized for controlling tumor growth, prolonging survival time, and improving quality of life to some extent. However, these therapies either alone or in combination have been shown to have numerous limitations and drawbacks: (i) Given poor diagnosis and other factors, most cancer patients are diagnosed too late to undergo surgery; even with the surgical indications, a series of complications might occur postoperatively such as bleeding, infection, bile reflux, and lymphedema, and postoperative recurrence and metastasis is quite common in patients who have had a resection. (ii) Chemotherapy and radiotherapy are still major postoperative adjunctive therapies or preferred therapies for patients with malignant tumors in middle and advanced stages, however, there are many side effects and complications such as myelosuppression, gastrointestinal tract reaction, cardiac damage, liver and renal function, or local radiation damage; in addition, cancer cells have ability to develop resistance to these conventional therapeutics over time and some cancers are insensitive to chemotherapy or radiotherapy. (iii) Targeted therapy as a newer type of cancer treatment which can more precisely identify and attack cancer cells, however, some drugs target substances that are more common on cancer cells but are also found on healthy cells and can affect healthy cells, too, causing side effects (e.g., high blood pressure, damage to liver, kidneys, or heart, allergic reactions) (3); in addition, the price of targeted drugs is so high that most patients cannot afford them. (iv) Immunotherapy is another new approach including cytokine infusions, cancer vaccines, and $\mathrm{T}$ cell therapy. It can stimulate immune cells to enhance their anticancer activity (4); however, the advantages of immunotherapy on improving the patient's quality of life and prolonging survival time are still unclear currently; furthermore, in addition the price of immunotherapy is so high that most patients cannot afford it. Therefore, more effective or adjunctive therapies must be soon developed for cancer prevention and treatment.

With development of medicine and update of knowledge, cancer therapy has come into a diversified comprehensive treatment stage. Many scholars put forward the concept of "survival with cancer", and they insist controlling cancer and causing cancer cells to "be static" and "hibernate" for a long time, is better than striving to reduce the lump and completely kill all cancer cells (5). In the process of "survival with cancer", traditional Chinese medicine (TCM) might play an important role.

$\mathrm{TCM}$, as an important component of complementary and alternative medicine, evolved over thousands of years with its own unique system of theories, diagnostics and therapies in Asian countries, especially China. In the world including Western countries, TCM has been increasingly used in the last decades and has become well known for its significant role in preventing and treating cancer. It is estimated that the United States National Cancer Institute (NCI) spends around \$120 million each year on TCM related research projects (6). According to conventional views, we believe that TCM possesses advantages as an adjuvant therapy to alleviate cancer symptoms at terminal stages when Western medicine treatments cannot offer any other treatment options. However, recent studies indicate that TCM can play an important role in the whole course of cancer prevention and treatment such as recovery stages post-operation, and when undergoing radiotherapy or chemotherapy (7). It may be capable of preventing tumorigenesis, shrinking or stabilizing tumors, reducing tumor recurrence and metastasis. It may also be capable of protecting cancer patients from suffering from complications, increasing sensitivity or reducing side-effects of conventional treatment, and improving quality of life and survival (8).

Since cancer is defined as a chronic disease like hypertension and diabetes, the role of TCM in treating chronic diseases especially cancer should not be overlooked. Thus, an understanding of TCM is needed by physicians and other health care providers. In this review, we will summarize the current evidence for using TCM as adjuvant cancer treatment in different stages of cancer lesions. First, some single TCMs and traditional herbal formulations which are commonly prescribed by traditional Chinese physicians for cancer patients will be summarized. Second, some TCM preparations which possess properties such as anti-cancer, improving immunity and protecting bone marrow, and are commonly used in clinical cancer treatment will be summarized. Third, the advantages of TCMs in different stages of cancer treatment such as recovery stages post-operation, and when undergoing radiotherapy or chemotherapy will be summarized. In a word, we hope this review should contribute to an understanding of TCM as adjuvant treatment for different stages of cancer, providing useful information for development of more effective anti-cancer drugs and making more patients "survival with cancer" for a long time.

\section{Single TCMs commonly prescribed by traditional Chinese physicians for cancer treatment}

Based on traditional Chinese medicine classic theory, the formation of tumors are usually due to deficiency of vital energy or Qi and blood in the body, combined with some pathogenic factors such as external evil invading, emotional abnormality, overeating and so on, leading to Qi stagnation, blood stasis and heat- and dampnessinduced toxicity blocking in the body, and then forming a lump or lumps for many days $(9,10)$. According to the above theory, TCMs which are used for cancer treatment usually fall into three categories: the first one with the properties of spiriting vital energy (Qi and blood), the second one with the properties of promoting blood 
circulation and removing blood stasis, and the third with properties of clearing heat and detoxifying (11). Numerous studies have shown that many TCMs (e.g., Ginseng Radix, Radix astragali, Radix Codonopsis, and Poria cocos) with properties of spiriting vital energy play important roles in cancer treatment with the basis summarized as follows: ( $i$ ) improving immune system function; (ii) protecting hematopoiesis of bone marrow; (iii) harmonizing gastrointestinal function $(12,13)$. In addition, numerous studies have shown that many TCMs with properties of promoting blood circulation and removing blood stasis (e.g., Angelica sinensis Radix, and Curcuma longa), or clearing heat and detoxifying (e.g., Hedyotis diffusa willd, and Scutellaria barbata), also play important roles in cancer treatment involving antiproliferation, anti-inflammation, anti-oxidation, antiangiogenesis, anti-thrombotic, immune-modulation and so on $(7,11,12,14)$. Thus, according to references and our clinical experience, we will choose some single TCMs commonly prescribed by traditional Chinese physicians for cancer treatment and give them a brief introduction especially regarding clinical studies (Table 1).

\subsection{Some single TCMs with properties of spiriting vital energy}

\subsubsection{Ginseng Radix}

Ginseng Radix (Ren-Shen in Chinese or Ginseng in Korea) is a well-known and popular TCM, which is believed to be the king of the herbs in the Orient particularly in China, Korea and Japan. It has been used for several thousand years with mysterious powers as a tonic, prophylactic and restorative agent (15). Ginseng Radix is reported to contain many active constituents including ginsenosides, essential oils, peptidoglycans, polysaccharides, nitrogen-containing compounds, fatty acids and phenolic compounds (16). Modern pharmacological studies have shown that Ginseng Radix and its active constituents have antitumor, antioxidant, immunomodulation, anti-ulcer, anti-adhesive, antioxidant, hepatoprotective, hypoglycemic activities, and so on (17). They have been proposed to have a chemopreventive effect on lung, gastric, liver, pancreas, and colon cancers and so on in cells, animals or humans (17-19). An epidemiological study indicated that patients taking ginseng had a $50 \%$ lower risk of cancer recurrence compared to patients not taking ginseng (12). In the following, we emphasize clinical research in recent years. An observational pilot study of cultivated wild Ginseng pharmaco-puncture (CWGP) was conducted at the East-West Cancer Center of Daejeon University (Daejeon, Korea) and the results indicated that CWGP showed potential as an effective treatment for advanced cancer patients on improving response and survival rate (20). A phase III trial was developed by Mayo Clinic Rochester (Rochester, USA) to evaluate the efficacy of Ginseng on cancer-related fatigue and the results indicated that $2,000 \mathrm{mg} /$ daily of Ginseng showed great benefit for ameliorating cancer-related fatigue without any discernible toxicity (21). Moreover, Ginseng extract could help in protecting tissue damage from inflammatory cytokines (IL-2, IL-10, IL-12, TNF-alpha, and IFN-gamma) in children after chemotherapy, which might be associated with decreased late complications of childhood (22). In addition, Ginseng appears to be a promising radio-protector and is capable of attenuating the deleterious effects of radiation on normal human tissue, and especially for cancer patients undergoing radiotherapy, which might be associated with its antioxidation and immunomodulation properties (23).

\subsubsection{Radix astragali}

Radix Astragali (Huang-Qi in Chinese), is the dried root of Astragalus membranaceus Bge. Var. mongholicus, and one of the most famous and frequently used herbal medicines and healthy food supplements used as a tonic. It has been used for over 2000 years in TCM prescriptions for the treatment of animal bites and poisons, wounds and burns, nephritis, diabetes, albuminuria, hypertension, cirrhosis, and various cancers (24). Chemical constituent investigations on Radix Astragali indicated that it contains several bioactive constituents including, isoflavonoids, triterpenoid saponins, polysaccharides, amino butyric acids and various trace elements; of which triterpenoid saponins represent the major beneficial constituents responsible for the bioactivities and efficacies of Radix Astragali on human health (25). Modern pharmacological studies have shown that Radix Astragali and its active constituents possess antioxidant, antitumour, hepatoprotective, anti-diabetic, antimicrobial, antiviral and immune enhancement activities $(25,26)$. They have been proposed to have an anticancer effect on breast, gastric, liver, colon, lung cancers and so on in cells, animals or humans. Via inhibiting cancer cell proliferation, regulating immunity, suppressing angiogenesis, or reducing side effects of chemotherapeutics, they play important roles in cancer therapy (26-28). In the following, we emphasize clinical research in recent years. Some studies indicated that decoctions of Huang-Qi compounds could reduce the proportion of patients who experienced nausea and vomiting and decrease the rate of leucopenia in colorectal cancer patients treated with chemotherapy. Huang-Qi compounds are also associated with increases in the proportions of T-lymphocyte subsets (CD3, CD4 and CD8). That is to say, HuangQi compounds may play important roles in stimulating immunocompetent cells and decreasing side effects in patients treated with chemotherapy (29). In addition, to investigate the effect of Huang-Qi injection on shortterm prognosis in childhood with acute lymphoblastic leukemia (ALL), a retrospective analysis was performed 


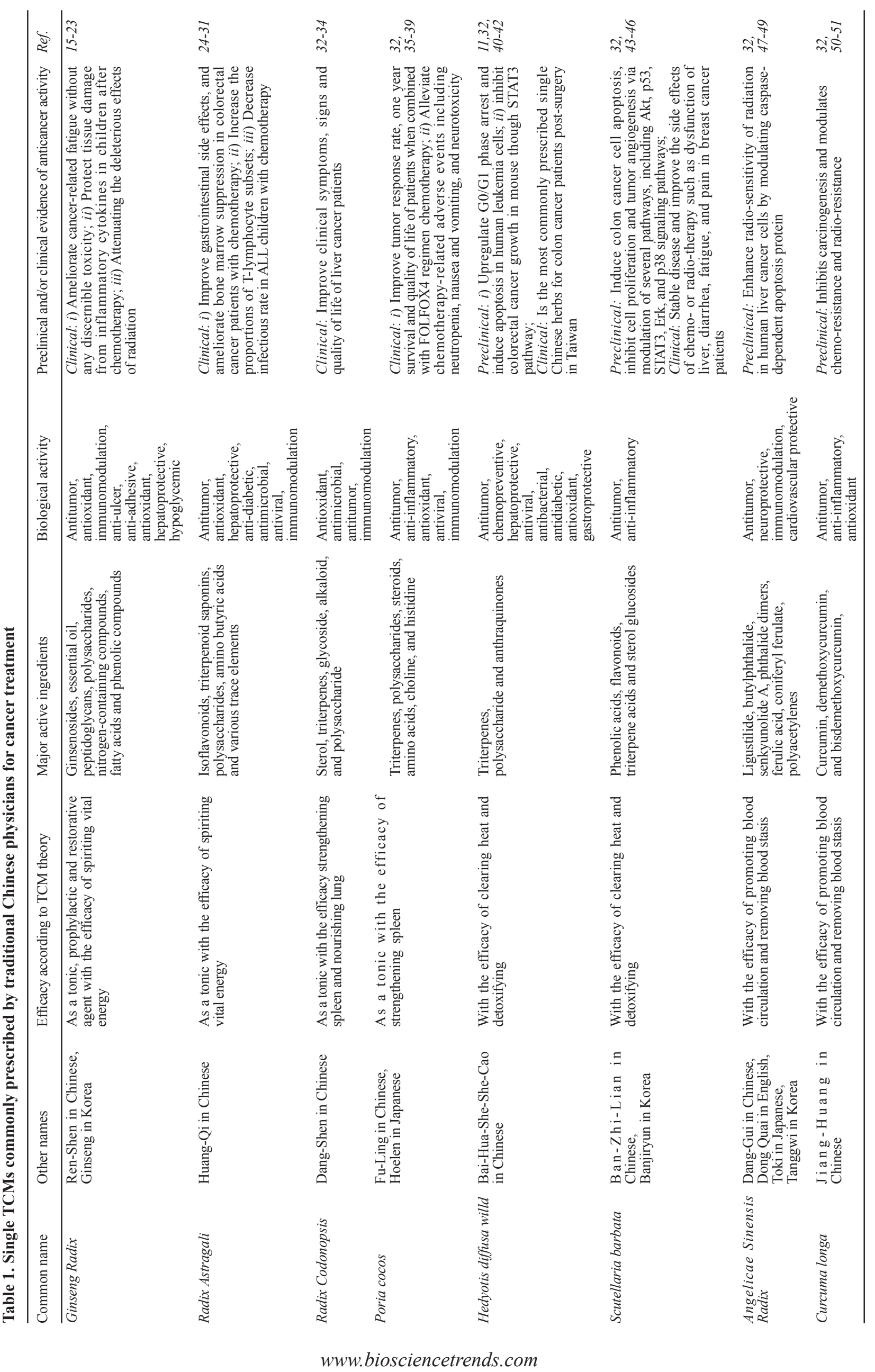


on the clinical data of 105 children newly diagnosed with ALL. The results indicated that Huang-Qi injection combined with chemotherapy had an enhanced antitumor effect and could improve the short-term prognosis and clinical outcome in children with ALL. Huang-Qi injection could reduce bone marrow suppression induced by chemotherapy or irritation of hematopoiesis of bone marrow and increase granulocyte level, and then decrease infectious rate in ALL children (30). In addition, it was reported that some TCM decoction of which HuangQi was a main ingredient could effectively prevent and reduce the occurrence and intensity of acute peripheral neuro-sensory toxicity caused by oxaliplatin (31).

\subsubsection{Radix Codonopsis}

Radix Codonopsis (Dang-Shen in Chinese) is the dried root of Codonopsis pilosula (Franch.) Nannf., and belongs to the family Campanulaceae. As one of the most popular TCMs in China, Japan and Korea, Radix Codonopsis has been used for thousands of years for treatment of dyspepsia, poor appetite, fatigue and psychoneurosis with properties of making the middle warmer, invigorating the spleen and nourishing the lung according to TCM theory (32). In many case, it was utilized primarily as a substitute of the much more costly Panax ginseng and was therefore called the poor man's ginseng. Some reports indicated that the main bioactive constituents were sterols, triterpenes, glycosides, alkaloids, polysaccharides and so on. Modern pharmacology research indicated that Radix Codonopsis and its active constituents had the functions of antioxidant, antimicrobial, antitumor and improving immunity (33). According to basic and clinical studies, they were reported to play important roles in cancer therapy via inhibiting cancer growth, regulating immunity, suppressing invasion and migration, or reducing side effects of chemotherapeutics. Radix Codonopsis could induce spleen lymphocyte proliferation, inhibit the decline of IL-2 levels in serum, and improve immune function in mice. Polysaccharides from Radix Codonopsis exhibited significant inhibitory effects on tumor cell growth, invasion, and migration of human epithelial ovarian cancer cells (34). Although Radix Codonopsis is commonly prescribed by traditional Chinese physicians for cancer treatment in the clinic, there are few clinical studies published currently in English. There was a report that some TCM decoction of which Radix Codonopsis was a main ingredient could effectively improve clinical symptoms, signs and quality of life of liver cancer patients. Thus, more rigorous trials are needed to confirm the efficacy of Radix Codonopsis and its active constituents on cancer therapy in the future.

\subsubsection{Poria cocos}

Poria cocos (Fu-Ling in Chinese or Hoelen in Japanese), is a kind of edible and pharmaceutical mushroom, and usually grows around the roots of old, dead pine trees. It is the dried sclerotium of the fungus Poria cocos (Schw.) Wolf (Fam. Polyporaceae). It is a wellknown TCM used to treat diabetes, dysentery, chronic fatigue syndrome, diarrhea, dizziness, edema, insomnia, kidney problems, nervousness, urination problems, and weakness (32). According to the record of the Chinese Pharmacopoeia (2010 edition), approximately $10 \%$ of TCM preparations contain Poria cocos. The chemical composition of Poria cocos mainly includes triterpenes, polysaccharides, steroids, amino acids, choline, histidine, etc (35). Modern pharmacological studies showed that Poria cocos and some of its active constituents had functions of anticancer, anti-inflammatory, antioxidant, antiviral and improved immunity $(35,36)$. According to basic and clinical studies, Poria cocos and some of its active constituents were reported to play important roles in cancer therapy via inhibiting cancer growth, regulating immunity, suppressing invasion and migration, or reducing side effects of chemotherapeutics in leukemia, lung, colorectal cancers and so on (37-39). Triterpenes from Poria cocos could induce apoptosis of leukemia (HL60) and lung (A549) cells via mitochondrial or death receptor pathways (37). Triterpenes from Poria cocos also could suppress growth and invasiveness of pancreatic cancer cells through the downregulation of MMP-7 (38). In addition, a systematic review evaluated clinical evidence for addition of herbal medicines to the FOLFOX 4 regimen chemotherapy for advanced colorectal cancer in the clinic. The results indicated that Poria cocos as one of the most frequently used herbs combined with the FOLFOX4 regimen chemotherapy could effectively improve tumor response rate, one year survival and quality of life of patients. It also could alleviate chemotherapy-related adverse events including neutropenia, nausea and vomiting, and neurotoxicity, compared to the FOLFOX4 regimen chemotherapy alone (39).

\subsection{Some single TCMs with properties of clearing heat and detoxifying}

\subsubsection{Hedyotis diffusa willd}

Hedyotis diffusa willd (Bai-Hua-She-She-Cao in Chinese) has been known as an ingredient of popular herbal teas and a famous TCM for a long time in the Orient and tropical Asia. Three major classes of compounds, including triterpenes, polysaccharides and anthraquinones, have been reported as the main bioactive compounds from this herb (32). Hedyotis diffusa willd and some of its active constituents have a wide variety of reported pharmacological activities, including anticancer, chemopreventive, hepatoprotective, antiviral, antibacterial, antidiabetic, antioxidant, and gastroprotective properties (40). It is widely applied in 
the treatment of inflammations such as appendicitis, urethritis, and bronchitis, hepatitis, tonsillitis, and sore throat due to its antibacterial activity (32). Recently, Hedyotis diffusa willd and some of its active constituents have gained increasing attention as an antitumor herb in liver, lung, prostate, colon, brain, pancreas and other cancers (40). The ethanol extract of Hedyotis diffusa willd could upregulate G0/G1 phase arrest and induce apoptosis in human leukemia cells by modulating caspase cascade signaling and altering the gene levels related to cell growth, signal transduction, apoptosis, cell adhesion, cell cycle, DNA damage and repair, transcription and translation by cDNA microarrays (41). The ethanol extract of Hedyotis diffusa willd also could inhibit colorectal cancer growth in mice though the STAT3 pathway without apparent adverse effects (42). In addition, a cross-sectional study on the prescription patterns and reasons for the use of TCM for colon cancer patients post-surgery in Taiwan were analyzed and the results indicated that Hedyotis diffusa willd was the most commonly prescribed single Chinese herbs for colon cancer (11). However, more rigorous trials are needed to confirm the efficacy of Hedyotis diffusa willd and its active constituents on cancer therapy in the future.

\subsubsection{Scutellaria barbata}

Scutellaria barbata (Ban-Zhi-Lian in Chinese or Banjiryun in Korea) is a perennial herb which is natively distributed throughout Korea and southern China and as a well-known herb commonly used in folk medicines. It has been used as an anti-inflammatory and an antitumor agent to treat hepatitis, liver cirrhosis, pulmonary abscess, appendicitis, osteomyelitis, hematemesis, epistaxis, dysentery, jaundice, sore throat, carbuncle, scrofula and malignant tumors for many years. The chemical composition of Scutellaria barbata mainly includes phenolic acids, flavonoids, triterpene acids and sterol glucosides (32). Recently, Scutellaria barbata and its active constituents have gained increasing attention for its use as an anti-tumor herb in human breast cancer, prostate cancer, leukemia, hepatoma carcinoma, uterine carcinoma, cervical carcinoma, lung carcinoma, skin cancer, colorectal carcinoma, renal adenocarcinoma, nasopharyngeal carcinoma and oral epidermoid carcinoma (43). On the basis of cDNA microarray analysis, the mechanism underlying the anticancer activity of Scutellaria barbata appears to involve DNA damage, cell cycle control, nucleic acid binding, protein phosphorylation and dephosphorylation, and dendritic cell functions (44). The ethanol extract of Scutellaria barbata was able to induce colon cancer cell apoptosis, inhibit cell proliferation and tumor angiogenesis via modulation of several pathways, including Hedgehog, Akt, p53, STAT3, Erk, and p38 signaling pathways and alteration of the expression of multiple critical target genes such as, Bcl-2, Bax, Cyclin D1, CDK4, and p21 in in vitro and in vivo studies (45). In addition, a phase 1B dose escalation trial of Scutellaria barbata (BZL101) for patients with metastatic breast cancer was conducted by Memorial Cancer Institute (Hollywood, FL, USA). The results indicated that oral administration of BZL101 was safe, well tolerated, and showed promising clinical evidence of anticancer activity in patients. It could effectively stabilize disease and improve the side effects of chemo- or radio-therapy such as dysfunction of liver, diarrhea, fatigue, and pain (40).

\subsection{Some single TCMs with properties of promoting blood circulation and removing blood stasis}

\subsubsection{SAngelica sinensis Radix}

Angelicae Sinensis Radix (Dang-Gui in Chinese, Dong Quai in English, Toki in Japanese, or Tanggwi in Korea), is the root of Angelica sinensis (Oliv.) Diel and has been used for thousands of years worldwide. Since, it has both properties of nourishing blood and promoting blood circulation and removing blood stasis, we will give it a brief introduction in this part. It is usually used to strengthen heart, lung, and liver meridians, as well as lubricate the bowel (32). Furthermore, it is named "female ginseng" because of its use for various health conditions of women including dysmenorrhea, pelvic pain, recovery from childbirth and menopausal symptoms (47). Over 70 compounds have been identified from Angelicae Sinensis Radix, including essential oils such as ligustilide, butylphthalide and senkyunolide A, phthalide dimers, organic acids and their esters such as ferulic acid, coniferyl ferulate, polyacetylenes, vitamins and amino acids (48). Angelicae Sinensis Radix and some of its active constituents have a wide variety of reported pharmacological activities, including antitumor, neuroprotective, immunomodulatory and cardiovascular protective functions (47). Recently, some reports indicated that Angelicae Sinensis Radix and some of its active constituents (e.g., Z-Ligustilide) exhibited great anticancer effects in liver, prostate, and oral cancers via inducing apoptosis, revising multidrug resistance or modulating lymphocyte activity and improving immunity (47-49). In addition, the decoction containing Radix Angelicae Sinensis could enhance radiosensitivity of radiation in human liver cancer cells by modulating caspase-dependent apoptosis protein (49). Although Radix Angelicae Sinensis is commonly prescribed by traditional Chinese physicians for cancer treatment in the clinic, there are few clinical studies published currently in English. Thus, more rigorous trials are needed to confirm the efficacy of Radix Angelicae Sinensis and its active constituents on cancer therapy in the future.

\subsubsection{Curcuma longa}

Curcuma longa (Jiang-Huang in Chinese), a member 
of the ginger family and commonly known as turmeric, is a culinary spice and therapeutic used in Asia for thousands of years to induce color and flavor in food as well as to treat a wide array of diseases such as diabetes, atherosclerosis, acne, jaundice, dysmenorrheal, as well as, cancer (32). Some reports indicated that among all spices, Curcuma longa has been proven for its better anticancer potential. Curcuminoids, for example, curcumin, demethoxycurcumin, and bisdemethoxycurcumin, are major components of Curcuma longa and they are reported to have numerous pharmacological activities including anti-inflammatory, antioxidant, and anticancer properties. Curcumin, a yellow natural polyphenol, is traditionally used as a spice and coloring in foods and is an important ingredient in curry. Recently, among curcuminoids, the anticancer properties of curcumin have been drawn more attention from researchers. Many studies indicated that its anticancer molecular mechanisms involved cell cycle arrest, angiogenesis and metastasis by regulating molecules such as, Cdk inhibitor, p21/WAF/CIPI, p53, NF $\kappa$ B, STAT-3, c-myc, COX-2, NOS, Cyclin D1, TNF $\alpha$, MMP-9, bFGF, EGF, GCSF, IL-8, PDGF, TGF $\alpha$, TNF, VEGF, fibronectin, vitronectin, and collagen. It also has a synergistic effect in combination with chemotherapeutics like cyclophosphamide, doxorubicin, mitomycin, etc. (50). In addition, extensive preclinical research within the last decade in cell culture and in animals has revealed that curcumin can sensitize tumors to different chemotherapeutic agents including doxorubicin, 5-FU, paclitaxel, vincristine, oxaliplatin, etoposide and so on in numerous cancers (e.g., breast, colon, pancreas, gastric, liver, blood, lung, prostate, and ovary) (51). Similar studies have also revealed that curcumin sensitizes a variety of tumors including, glioma, neuroblastoma, cervical carcinoma, epidermal carcinoma, prostate cancer, and colon cancer to radiotherapy. Moreover, curcumin has also been shown to protect normal organs such as liver, kidney, oral mucosa, and heart from chemotherapy and radiotherapyinduced toxicity. However, there are few clinical studies about Curcuma longa and its active compound curcumin in cancer therapy published currently in English. Thus, more rigorous trials are needed to confirm the efficacy of Curcuma longa and its active constituents on cancer therapy in the future.

\section{Traditional Chinese herbal formulations commonly prescribed by traditional Chinese physicians for cancer treatment}

Traditional Chinese herbal formulations, or Kampo in Japanese, are a combination of compatible herbs in fixed dosages, most of which come from classical or well known Chinese textbooks of medicine like "Shang Han Lun" and "Jin Gui Yao Lue" (52). Currently, several traditional Chinese herbal formulations, such as Bu-zhong-yi-qi-tang, have been found to have a potentially beneficial effect for treating various cancers. A brief outline of the anticancer pharmacology of some traditional Chinese herbal formulations commonly prescribed by traditional Chinese physicians for cancer treatment is presented below (Table 2).

\subsection{Bu-zhong-yi-qi-tang}

Bu-zhong-yi-qi-tang (Hochuekki-to or TJ-41 in Japanese, or Bojungikki-Tang in Korean) is a classical formulation widely used in China, Japan, and South Korea for a long time. It was found by Dongyuan Li (A.D. 1,180-1,251, Jin and Yuan dynasties) as a tonic for the treatment of weakness including fatigue, visceroptosis, gastrointestinal motility disorder, and rectal prolapse due to chronic diarrhea and has been identified as an effective drug for the treatment of TCM spleen-qi deficiency in clinical practice. It contains 7 herbs including Pinellia tuber, Scutellaria baicalensis, Zingiberis rhizoma, Zizyphi fructus, Coptidis rhizoma, Glycyrrhiza radix, and Panax ginseng (12). Recently, much of the pharmacological research has shown that Bu-zhong-yiqi-tang has potent immunomodulatory and anticancer properties. TJ-41 has a significant chemo-preventative effect on ovarian and liver cancer lines by inducing apoptosis or arresting the cell cycle (12). It could restore mitomycin $\mathrm{C}$ (MMC)-induced immunosuppression in mice by increasing the activity of bone-marrow cells and natural killer (NK) cells and preventing lethal HSV-1 infection (53). Preoperative administration of $\mathrm{TJ}-41$ in patients with gastrointestinal malignancies showed that it could prevent surgical stress-induced immunosuppression by maintaining NK cell activity and inhibiting the elevation of stress mediators noradrenaline and IL-6 (54). In addition, TJ-41 might have beneficial effects on cancer-related fatigue and quality of life in cancer patients, and it also could reduce the extent of side effects such as leucopenia and intestinal damage and fatigue occurring as a result of radiation or chemotherapy to treat malignant tumors $(12,55)$.

\subsection{Shi-quan-da-bu-tang}

Shi-quan-da-bu-tang (Juzentaiho-to or TJ-48 in Japanese) is a well-known Chinese herbal formulation first recorded in the Chinese Song Dynasty (about A.D. 1,200) and it comprises 10 herbs including Ginseng radix, Astragali radix, Angelicae radix, Rehmanniae radix, Atractylodis lanceae rhizoma, Cinnamomi cortex, Poria, Paeoniae radix, Ligustici rhizoma and Glycyrrhizae radix (12). It has been used for many years for the treatment of various kinds of disease such as anemia, rheumatoid arthritis, atopic dermatitis, chronic fatigue syndrome, and ulcerative colitis. Recently, TJ-48 has been reported to have antitumor effects and to modulate immune responses. It could reduce the side effects of 


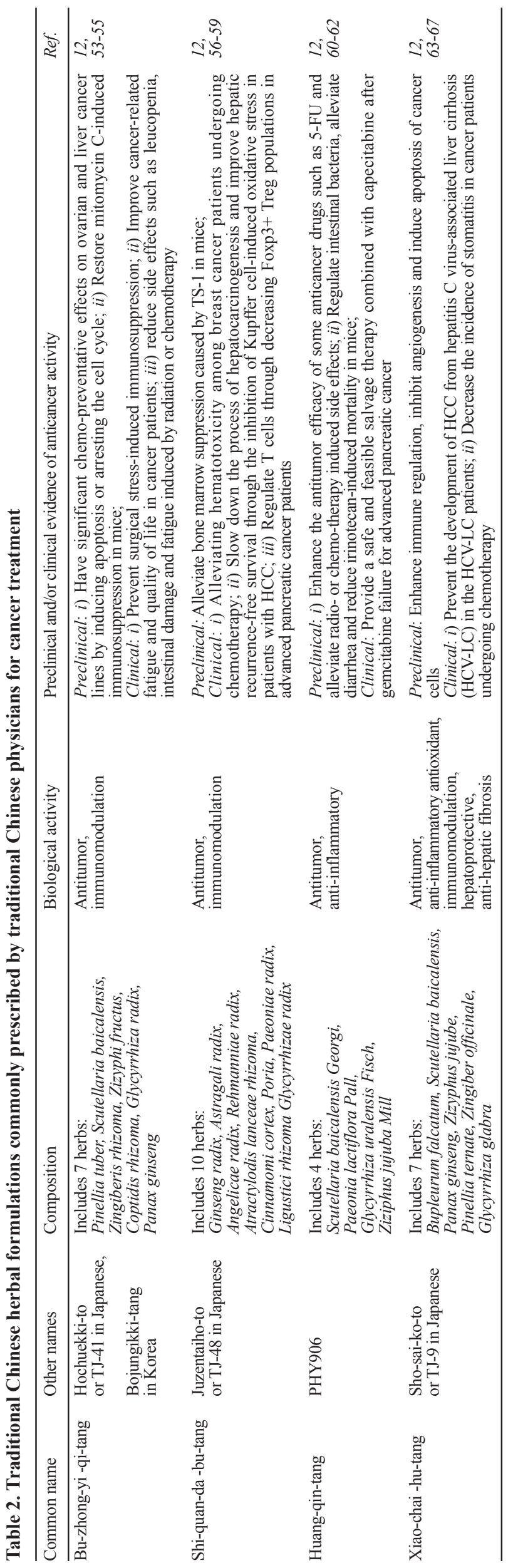

chemotherapy, radiation therapy and surgical treatment, and prevent various types of cancers (e.g., breast, liver, brain and pancreatic cancer) or their metastasis according to numerous preclinical and clinical studies. TJ-48 was effective in alleviating bone marrow suppression caused by TS-1 (an oral anticancer drug containing a 5 -fluorouracil derivative Tegafur) in mice (56). TJ-48 was also effective in alleviating hematotoxicity among patients with breast carcinoma receiving chemotherapy, without affecting the presentation of tumor markers (CEA and CA153) in the short term (57). Moreover, TJ48 could slow down the process of hepatocarcinogenesis and improve hepatic recurrence-free survival through the inhibition of Kupffer cell-induced oxidative stress in patients with hepatocellular carcinoma (HCC) (58). TJ-48 also could increase regulatory activities in T cells through decreasing Foxp $3^{+}$Treg populations in advanced pancreatic cancer patients, and this effect might lead to immune-augumentation for various combination therapies (59).

\subsection{Huang-qin-tang}

Huang-qin-tang is a classical traditional Chinese herbal formulation with four herbs (Scutellaria baicalensis Georgi, Paeonia lactiflora Pall, Glycyrrhiza uralensis Fisch, and Ziziphus jujuba Mill), and it was first recorded in "Shang Han Lun" which is one of the famous classics of TCM edited by a well-known Chinese physician during the Han Dynasty Zhongjing Zhang. It has been used for over 1800 years to treat a variety of gastrointestinal symptoms including diarrhea, nausea and vomiting, and abdominal cramps (12,60). PHY906 is a modified pharmaceutical preparation derived from the traditional herbal formulation Huang-qin-tang and it consists of the same four herbs as Huang-qin-tang at a relative weight ratio of 3:2:2:2 (12). To ensure standardization and maintain inter-batch reliability of PHY906, high performance liquid chromatography (HPLC) was used to establish a "chemical fingerprint" of PHY906 by Professor Yung-Chi Cheng and his team (Yale University School of Medicine). Furthermore, they have conducted a series of preclinical and clinical studies to investigate the anticancer activities of PHY906 in recent years. In vivo studies, the combination of PHY906 with irinotecan, capecitabine, 5-FU, and leucovorin (LV) resulted in significant improvement in gastrointestinal toxicities, antitumor activity, and overall survival with no increased host toxicity versus chemotherapeutics alone (60). In the case of irinotecan, for which severe delayed-onset diarrhea is the major dose-limiting toxicity, PHY906 could effectively regulate intestinal bacteria, alleviate diarrhea and reduce drug-induced mortality in mice. Moreover, treatment with PHY906 mitigated the intestinal injuries of fractionated whole abdomen irradiation and improved recovery from radiation injury, which indicated PHY906 
as a potential adjunct to radiation therapy (61). In addition, a phase II study was conducted to explore the efficacy of capecitabine combined with PHY906 in patients with advanced pancreatic cancer who were previously treated with gemcitabine-based regimens. The results showed capecitabine plus PHY906 provided a safe and feasible salvage therapy after gemcitabine failure for advanced pancreatic cancer with six-month survival rate at $44 \%$ (62).

\subsection{Xiao-chai-hu-tang}

Xiao-chai-hu-tang (Sho-sai-ko-to or TJ-9 in Japanese), a classical traditional Chinese herbal formulation originally recorded in "Shang Han Lun", has been used to treat liver diseases especially chronic hepatitis and liver cancer for thousands of years in China and Japan. It consists of seven medicinal herbs (Bupleurum falcatum, Scutellaria baicalensis, Panax ginseng, Zizyphus jujube, Pinellia ternate, Zingiber officinale, and Glycyrrhiza glabra) (63). Much pharmacological research has shown that TJ-9 has potent antiinflammation, antioxidation, immunomodulation, hepatoprotective, anti-hepatic fibrosis, and antitumor properties. Recently, many basic or clinical studies have been conducted to assess the beneficial effects and safety of TJ-9 for cancer treatment. These studies have demonstrated that TJ-9 treats cancer by enhancing immune regulation, anti-angiogenesis and apoptosis of tumor cells (64). TJ-9 exhibited significant growth inhibition of ovarian cancer cell lines, and the mechanisms of the inhibitory effects can be attributed, in part, to apoptosis induced by TJ-9. TJ-9 could effectively inhibit the growth of $\mathrm{H} 22$ mouse solid liver cancer and improve the immune function of tumorbearing mice by increasing NK cells, T lymphocytes and IL-2 levels (65). Furthermore, TJ-9 plays important roles in preventing hepatocarcinogenesis. A study was performed to find a way to prevent the development of HCC from hepatitis $\mathrm{C}$ virus-associated liver cirrhosis (HCV-LC) in HCV-LC patients who had received reduction ALT therapy such as TJ-9. The results indicated that chances of surviving for more than ten years without developing HCC for HCV-LC patients (66). In addition, TJ-9 gargle as a gargling agent for patients receiving chemotherapy showed a significantly decreased incidence of stomatitis, and a painkilling effect compared to gargling with providone-iodine and amphotericin B. Thus, TJ-9 gargle was considered to be a useful method against stomatitis prevention and sharp pain mitigation from chemotherapy (67).

\section{Chinese medicine preparations commonly used in clinical practice for cancer treatment}

Chinese medicine preparations are a form of Chinese herbal medicine that are isolated from single herbs or their active compounds or herbal formulations and prepared using modern advanced pharmaceutical technology. There are various dosage forms including injections, tablets, pills, capsules, and liquids. Compared to traditional decoctions, Chinese medicine preparations are safer, more effective, and easier to use $(12,32)$. Thus, Chinese medicine preparations are becoming increasingly popular in China and are attracting worldwide attention.

Currently in China, some Chinese medicine preparations are derived from single TCMs or their active compounds or herbal formulations, which have the properties of spiriting vital energy and their anticancer molecular mechanisms mainly by improving immunity (e.g., Shenqi fuzheng injection and Kanglaite injection). Some Chinese medicine preparations are derived from single TCMs or their active compounds or herbal formulations, which have properties of clearing heat and detoxifying, promoting blood circulation and removing blood stasis and their anticancer molecular mechanisms involving apoptosis, cell cycle arrest, angiogenesis and metastasis, immunoregulation and so on (e.g., Huachansu injection and Cantharidin sodium injection). We want to stress that some TCMs and some natural compounds like Mylabris, Chansu, camptothecin derivatives, and vinca alkaloids are toxic. However, the application of these toxicants provides a magic power to deal with severe diseases like cancer, and this process might be described as "fighting fire with fire" (68). Thus, in the following, a brief outline of the oncologic pharmacology of the most commonly used Chinese medicine preparations including some toxicants that have been approved by the State Food and Drug Administration (FDA) of China are briefly presented below (Table 3 ).

\subsection{Shenqi fuzheng injection}

Shenqi fuzheng injection is an injectable traditional Chinese herbal formula comprised of two herbal medicines, Radix Astragali (Huang-Qi) and Codonopsis pilosula (Dang-Shen). The injection has been approved by China's FDA since the 1990s. It is commonly used to improve immune function against chronic diseases including cancer and cerebrovascular diseases such as, angina, coronary heart disease, heart failure, and so on (32). Recently, many trials have demonstrated that Shenqi fuzheng injection might play an important role in the treatment of various advanced cancers. It could improve immune response via raising activity of NK cell, macrophage and T-lymphocyte subgroups without any injuries of heart, liver and kidney function or other adverse reactions in cancers. It also could reduce the toxicity of radiation therapy and chemotherapy. A systematic meta-analysis involving thirteen randomized controlled trials and 860 patients was conducted on Shenqi fuzheng injection for advanced gastric cancer (69). It showed that the combination of chemotherapy with Shenqi fuzheng injection obtained a series of positive results including improving quality of life, 
Table 3. Chinese medicine preparations commonly used in clinical for cancer treatment

\begin{tabular}{|c|c|c|c|c|}
\hline Common name & Source or composition & Biological activity & Preclinical and/or clinical evidence of anticancer activity & Ref. \\
\hline $\begin{array}{l}\text { Shenqi } \\
\text { fuzheng injection }\end{array}$ & $\begin{array}{l}\text { Comprised of } 2 \text { herbs: } \\
\text { Radix Astragali } \\
\text { Codonopsis pilosula }\end{array}$ & $\begin{array}{l}\text { Antitumor, } \\
\text { immunomodulation }\end{array}$ & $\begin{array}{l}\text { Clinical: Improve quality of life, increase complete remission and } \\
\text { partial remission efficacy rate, enhance immunity, and decrease } \\
\text { adverse events such as nausea, vomiting, oral mucositis, and } \\
\text { leucopenia in cancer patients undergoing chemotherapy }\end{array}$ & $\begin{array}{l}32, \\
69-71\end{array}$ \\
\hline $\begin{array}{l}\text { Kanglaite } \\
\text { injection }\end{array}$ & $\begin{array}{l}\text { Extracted from } \\
\text { Semen Coicis Yokuinin }\end{array}$ & Antitumor & $\begin{array}{l}\text { Clinical: } i \text { ) Improve the short-term efficacy, improve the quality } \\
\text { of life, and decrease the risk of gastrointestinal reaction and } \\
\text { myelosuppression in cancer patients undergoing radio- or chemo- } \\
\text { therapy; } i \text { ) combined with hepatic arterial intervention could } \\
\text { improve the short-term clinical efficacy, quality of life, and } \\
\text { decrease the pain of patients with unresectable HCC }\end{array}$ & $\begin{array}{l}12, \\
72-74\end{array}$ \\
\hline $\begin{array}{l}\text { Huachansu } \\
\text { injection }\end{array}$ & $\begin{array}{l}\text { Extracted from the skin } \\
\text { and parotid venom glands } \\
\text { of the toad Bufo bufo } \\
\text { gargarizans Cantor }\end{array}$ & $\begin{array}{l}\text { Antitumor, } \\
\text { Anti-HBV } \\
\text { immunomodulation }\end{array}$ & $\begin{array}{l}\text { Preclinical: Inhibit cell proliferation, induce cell differentiation } \\
\text { and apoptosis, disrupt cell cycle, inhibit cancer angiogenesis, } \\
\text { reverse multi-drug resistance, and regulate immune response in } \\
\text { cancer cells } \\
\text { Clinical: } i \text { ) Enhance the antitumor efficacy of gemcitabine and } \\
\text { oxaliplatin and improve the quality of life of patients; } i \text { i) Postpone } \\
\text { tumor recurrence and metastasis, prolong the survival time and } \\
\text { increase the survival rate of post-surgical patients with HCC; iii) } \\
\text { Decrease the quantity of pericardial effusion in cancer patient }\end{array}$ & $\begin{array}{l}12, \\
75-80\end{array}$ \\
\hline $\begin{array}{l}\text { Cantharidin } \\
\text { sodium injection }\end{array}$ & $\begin{array}{l}\text { Extracted from } \\
\text { blister beetles }\end{array}$ & $\begin{array}{l}\text { Antitumor, } \\
\text { immunomodulation }\end{array}$ & $\begin{array}{l}\text { Clinical: } i) \text { Improve quality of life; } i i) \text { Reduce side effects } \\
\text { induced by chemotherapy such as leukopenia and gastrointestinal } \\
\text { reactions }\end{array}$ & $81-83$ \\
\hline
\end{tabular}

increasing complete remission and partial remission efficacy rate, and decreasing adverse events such as nausea, vomiting, oral mucositis, and leucopenia. Moreover, Shenqi fuzheng injection intervention appeared to be useful to increase efficacy and reduce toxicity when combined with platinum-based chemotherapy for advanced non-small-cell lung cancer (NSCLC) and colorectal cancer $(12,70)$. In addition, the combination of concurrent chemoradiotherapy with Shenqi fuzheng injection in patients with head and neck neoplasms could effectively enhance immunity with the levels of CD3, CD4, and CD4/CD8 increased, improve quality of life, and decrease adverse events such as nausea, vomiting, oral mucositis, skin lesions, and leucopenia (71).

\subsection{Kanglaite injection}

Kanglaite injection is an anti-tumor drug, which contains extracts from Chinese herbal medicine coix seed (Semen Coicis Yokuinin) using modern advanced pharmaceutical technology. In August 1997, Phase III clinical trials were completed and Kanglaite injection was officially launched in China after final approval from the Ministry of Public Health (12). Kanglaite injection is mainly used for the treatment of NSCLC, liver cancer, gastric cancer, etc. It has been found to significantly decrease cancer cachexy, improve quality of life of cancer patients, and may ameliorate multiple drug resistance of cancers when combined with radiotherapy and chemotherapy in clinical use. A systematic meta-analysis involving 34 clinical trials was conducted to assess the effects of Kanglaite injection combined with chemotherapy versus chemotherapy alone in the treatment of advanced non-small cell lung carcinoma (72). It showed that the combination could improve the short-term efficacy, and performance status and decrease the risk of gastrointestinal reaction compared with systematic chemotherapy alone. Another systematic meta-analysis involving 9 clinical trials indicated that Kanglaite injection combined with hepatic arterial intervention could improve the short-term clinical efficacy, quality of life, and decrease the pain of patients with unresectable HCC (73). Moreover, a network of meta-analysis involving 38 randomized controlled trials and 2,761 participants was conducted to compare which was the best Chinese herb injection based on the FOLFOX regimen for gastric cancer. Kanglaite injection exhibited greater effects than many other Chinese herb injections in clinical efficacy and safety for gastric cancer. It could strengthen the overall response rate, improve the quality of life, reduce nausea and vomiting, and reduce the incidence of leukopenia (III-IV) (74).

\subsection{Huachansu injection}

Huachansu injection or Cinobufacini injection is a water-soluble preparation extracted from the skin and parotid venom glands of the toad (Bufo bufo gargarizans Cantor) which contains Chansu. It has been approved by China's FDA since the 1990s and widely used to treat patients with lung, liver, colon, and pancreatic cancers at oncology clinics in China (12). Cardiac glycosides including bufalin, resibufogenin, and cinobufagin are the three major active constituents to which the antitumor activity of Huachansu injection may be attributed. Huachansu injection exhibited significant effects on inhibition cell proliferation, induction of cell differentiation and apoptosis, disruption of the cell cycle, inhibition of cancer angiogenesis, reversal of multi-drug resistance, and regulation of the immune response in cancer cells $(75,76)$. It also could effectively 
enhance physical immunity and improve the quality of life with little toxicity in cancer patients. A pilot study of Huachansu injection in patients with HCC, NSCLC, and pancreatic cancer showed that Huachansu injection could improve the quality of life of patients and even enhanced tumor shrinkage with little toxicity (77). Another clinical study using Huachansu injection in combination with gemcitabine and oxaliplatin in treating gallbladder carcinomas showed that Huachansu injection substantially enhanced the antitumor efficacy of gemcitabine and oxaliplatin and improved the quality of life of patients (78). Moreover, a case-control trial $(n=120)$ was conducted to assess the effects of Huachansu injection plus Jiedu granules (a Chinese medicine compound) versus transcatheter arterial chemoembolization (TACE) in post-surgical patients with HCC in Changhai Hospital (Shanghai, China). Huachansu injection plus Jiedu granules could postpone tumor recurrence and metastasis, prolong survival time and increase survival rate of post-surgical patients with HCC (79). In addition, Huachansu injection has been reported to be effective for treating malignant pericardial effusion, pleural effusions, and ascites. Sun et al. reported a case of advanced lung cancer with malignant pericardial effusion treated by intrapericardial Huachansu injection instillation (80). Huachansu injection could effectively relieve the patient's cardiac tamponade symptoms and improve the patient's quality of life with the levels of CA-125 in pericardial effusion decreased and the quantity of pericardial effusion significantly reduced. Furthermore, there were little gastrointestinal adverse reactions and myelosuppression in the patient after injection of Huachansu in the pericardial cavity.

\subsection{Cantharidin sodium injection}

Cantharidin is a sesquiterpene derivative extracted from blister beetles which has long been used as a poisonous TCM to treat life-threatening diseases such as cancer according to the principle "fighting fire with fire". Cantharidin is a potent and selective inhibitor of protein phosphatase 2A (PP2A). Recent pharmacologic studies proved that cantharidin could induce cell cycle arrest and apoptosis, and interfere with the metabolism of nucleic acids and of proteins in cancer cells, significantly inhibit the growth of various cancers including HCC, uterine cervix cancer, nasopharyngeal carcinoma, cutaneous cancer, leucemia, and so on (81). Cantharidin sodium injection is a semi-synthetic derivative of cantharidin. It has been approved by China's FDA since the 1990s and widely used to treat patients with lung, liver, colon, pancreatic, gastric and breast cancers at oncology clinics in China. It could reduce the uptake of amino acids in cancer cells, inhibit protein synthesis, stimulate macrophages, lymphocytes, cause polymorphonuclear cells to produce interleukin, and finally improve immunity and enhance anticancer efficacy (82). Several clinical studies showed that Cantharidin sodium injection combined with chemotherapy could effectively improve quality of life and reduce side effects induced by chemotherapy such as leukopenia and gastrointestinal reactions without any increase in toxicity in patients with NSCLC, colorectal, breast and gastric cancer $(81,83)$. Although Cantharidin sodium injection is wildly used in cancer therapy in China, most of these studies are published in Chinese and little is known about use of the Cantharidin sodium injection outside of China. Thus, the mechanisms of the injection's action must be investigated and the injection must be clinically evaluated further.

\section{Clinical trials of TCMs as adjuvant treatment in the whole course of cancer therapy}

As is known, surgery, chemotherapy and radiotherapy are major conventional cancer therapies. Although these therapies are directed at killing or eradicating cancer cells, a series of complications and side effects will come along such as upper limb lymphedema, infection/fever, fatigue, pain, anemia, diarrhea, nausea and vomiting, hair loss, and bone marrow suppression. These complications and side effects inconvenience and cause discomfort to patients and they may also limit or prevent the delivery of therapy at its optimal dose and time, potentially causing fatalities. Thus, more effective therapies to help prevent and control complications and side effects of conventional cancer therapy must soon be developed. Some TCMs have been found to be adjunctive in cancer therapy. Here we will give a brief outline on the use of TCMs to reduce some complications and side effects associated with conventional cancer therapy in clinical studies (Table 4).

\subsection{Lymphedema}

Lymphedema is a serious medical complication commonly associated with breast cancer treatment, such as surgery and/or radiation, and it may occur more than 5 years after surgery. Lymphedema involves the accumulation of protein-rich fluid in the interstitial space, and occurs when the lymphatic system is damaged and no longer properly drains the lymph fluid back to the systemic circulation (84). When the lymphatic system is damaged, fluid accumulates in the affected limb, leading to swelling, fibrosis, reduced range of motion, decreased function, and, in later stages, infection and pain. However, it is difficult to cure once it occurs despite the various treatments which have been developed such as massage, compression garments, bandages, or sleeves, manual lymphatic drainage, individualized exercise, and education regarding skin care and infection prevention (85). In recent years, some reports related to the effectiveness of TCMs use among cancer patients with lymphedema have been reported.

Coumarin, a chemical compound derived from some 


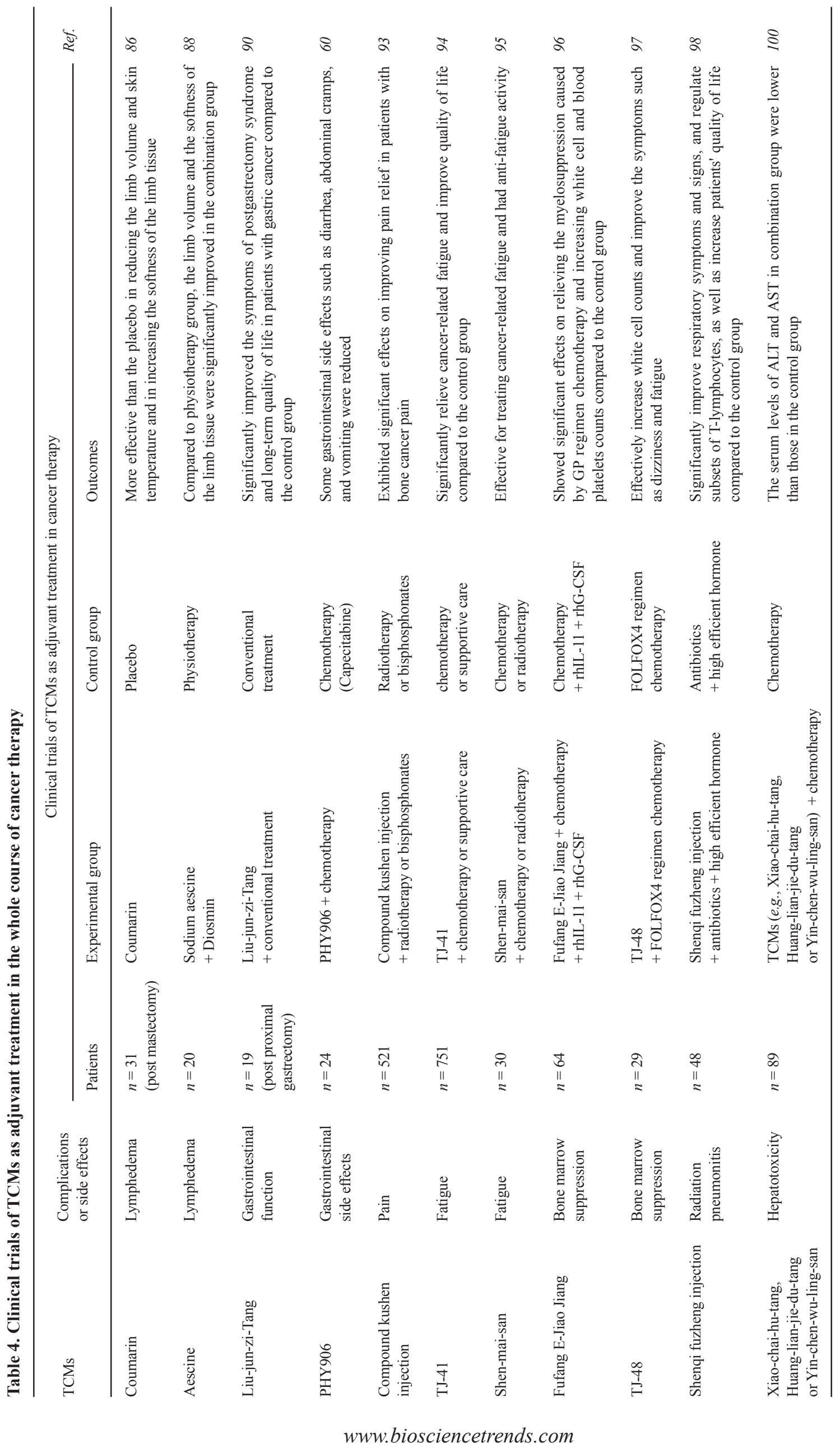


plants such as Tonka beans, might reduce lymphedema and the incidence of secondary infections because it decreases the volume of protein by stimulating proteolysis. A randomized, double-blind, placebocontrolled, cross-over trial to examine the effect of coumarin in 31 women with post-mastectomy lymphedema and 21 men and women with lymphedema of the lower extremity of various causes was conducted with the patients receiving $400 \mathrm{mg}$ of coumarin or placebo for 6 months. The results indicated that coumarin was more effective than the placebo in reducing the limb volume and skin temperature and in increasing the softness of the limb tissue, with mild side effects, such as mild nausea and diarrhea arising in seven patients who took coumarin (86). However, Loprinzi et al. reported that coumarin had potential negative and even life-threatening side effects, such as hepatotoxicity (87). Therefore, further research with a rigorous design and larger sample size is needed to re-evaluate the effectiveness of coumarin in treating lymphedema.

Sodium aescine is a Chinese medicine preparation extracted from Horsechestnut seed and is widely used to treat chronic venous insufficiency. It is also widely used to treat soft tissue swelling caused by various reasons such as breast cancer related upper limb lymphedema. To observe the efficacy of sodium aescine combined with Diosmin on upper limb lymphedema in patients after breast cancer surgery, a randomized controlled study was conducted. The results indicated that compared to physiotherapy group $(n=16)$, the limb volume and the softness of the limb tissue were significantly improved in the combination group $(n=20)(88)$. Moreover, a possible mechanism of aescine may be related to inhibiting the activity of enzymes elastase and hyaluronidase (85). However, there are several limitations associated with the current published studies on sodium aescine such as indeterminate results, small sample sizes, and little examination of outcomes. Therefore, further research with rigorous design and larger sample size is needed to re-evaluate the effectiveness of sodium aescine in treating lymphedema.

\subsection{Gastrointestinal side effects}

Gastrointestinal side effects including loss of appetite, diarrhea, nausea, and vomiting are the most common symptoms occurring in cancer patients after surgery and/ or receiving chemo- or radio-therapy. However, there is still no effective treatment to ameliorate these symptoms in cancer patients. Recently, many clinical trials have suggested that some TCMs may be effective at treating gastrointestinal side effects. Liu-jun-zi-Tang (or Rikkunshito in Japanese) is a famous Traditional Chinese herbal formulation including 6 herbs (Ginseng Radix, Poria cocos, Rhizoma atractylodis macrocephalae, liquorice root, pinelliae tuber, pericarpium citri, common ginger, and Jujube). It is widely used in China and Japan for the treatment of upper gastrointestinal symptoms of patients with functional dyspepsia, gastroesophageal reflux disease, dyspeptic symptoms of post-gastrointestinal surgery patients, and chemotherapyinduced dyspepsia in cancer patients. Some Japanese researchers found that Liu-jun-zi-tang as a ghrelin enhancer was effective for treating cisplatin-induced dyspepsia and cancer cachexia-anorexia syndrome (89). Liu-jun-zi-tang significantly improved the symptoms of postgastrectomy syndrome and long-term quality of life in patients with gastric cancer who had undergone proximal gastrectomy (90). In addition, some clinical studies have shown that PHY906 enhances the therapeutic indices of a broad spectrum of anticancer agents such as Capecitabine, 5-FU and irinotecan in colorectal, liver, and pancreatic cancers. PHY906 could reduce chemotherapy-induced toxicities especially gastrointestinal side effects (e.g., diarrhea, abdominal cramps, and vomiting) and/or increase chemotherapeutic efficacy without affecting the pharmacokinetics of chemotherapeutic agents $(12,60)$. Furthermore, advanced clinical trials are ongoing to demonstrate the effectiveness of PHY906 as adjuvant therapy for cancer patients undergoing chemotherapy.

\subsection{Pain}

Pain is a common and burdensome symptom of cancer and the causes of pain can be the cancer itself (the tumor pressed on bones, nerves, or other organs) or its treatment (e.g., surgery, chemotherapy, or radiotherapy). It was reported that $75-90 \%$ cancer patients especially who have bone metastasis experienced pain during their illness (91). As indicated in current WHO guidelines, three step analgesic ladder therapies are the standard of care for cancer pain. However, up to $50 \%$ of cancer pain is still undertreated. In recent years, many clinical trials have suggested that some TCMs as adjunctive therapy may be effective at treating cancer related pain and that their effects are similar to those of Western analgesics. TCMs may reduce the side effects of conventional analgesics, and then enhance cancer patients' quality of life (12). To assess the effectiveness of oral TCMs in relieving pain secondary to bone metastases in patients, a metaanalysis enrolled a total of 16 randomized controlled trials and 1,008 patients were identified and analyzed (92). The results showed that TCMs plus conventional treatment increased the pain-relief rate compared with the conventional treatment alone. Another meta-analysis enrolled a total of 7 randomized controlled trials and 521 patients were identified and analyzed to assess the efficacy and safety of compound kushen injection (a preparation extracted from Radix sophorae flavescentis) for bone cancer pain. Compared with radiotherapy or bisphosphonates, compound kushen injection showed significant effects on improving pain relief in patients with bone cancer pain (93). In addition, some clinical 
trials indicated that acupuncture might be beneficial for alleviating cancer pain. In all, TCM interventions appear to have beneficial effects on pain secondary to bone metastases in patients. However, there are several limitations associated with the current published studies on TCMs relieving pain such as indeterminate results, small sample sizes, and little examination of outcomes. Therefore, further research with rigorous design and larger sample size is needed to re-evaluate the effectiveness of TCMs in treating cancer related pain.

\subsection{Fatigue}

Cancer-related fatigue is a highly prevalent, persistent and subjective sense of tiredness related to cancer disease or cancer treatment which cannot be relieved by sleep or rest, and overall $50-90 \%$ of people with cancer experience fatigue. It significantly interferes with patients' daily activities and decreases their quality of life $(12,94)$. However, it remains under-recognized and under-treated, partly because of limited understanding of its pathophysiology and lack of effective treatments. Several Chinese herbal medicines may have beneficial effects on cancer-related fatigue and quality of life for cancer patients. To assess the effectiveness and safety of Chinese herbal medicine for the treatment of cancer-related fatigue, a meta-analysis enrolled a total of 10 randomized controlled trials and 751 patients with various cancers such as liver, lung, and breast cancer were identified and analyzed (94). The results showed that some TCMs such as TJ-41 used alone or in combination with chemotherapy or supportive care showed significant relief in cancer-related fatigue and improved quality of life compared to chemotherapy or supportive care based on single trials. In addition, a randomized, double-blind, placebo-controlled clinical trial was conducted to evaluate the efficacy of Shen-maisan (a famous Traditional Chinese herbal formulation composed of processed Ginseng, Liriope spicata, and Schizandrae fructus) in patients with cancer who were undergoing chemotherapy or radiotherapy (95). Shenmai-san was found to be effective for treating cancerrelated fatigue and had anti-fatigue activity. According to TCM theory, Shen-mai-san has a synergistic effect for qi and yin deficiency which are similar to chemotherapy- or radiotherapy-induced side effects and has the ability to prevent fatigue. These findings from a limited number of trials suggest that TCM seems to be effective and safe in the treatment of cancer-related fatigue. However, current evidence is insufficient to draw a confirmed conclusion due to the poor methodological quality of included trials. However, there are several limitations associated with the current published studies on TCMs relieving fatigue such as indeterminate results, small sample sizes, and little examination of outcomes. Thus, conducting rigorously designed trials on potential Chinese herbal medicine are warranted.

\subsection{Bone marrow suppression}

Bone marrow suppression is a reduction in the activity of bone marrow, resulting in decreased numbers of red blood cells, platelets, and white blood cells. One of the most common reasons for a patient to have this condition is chemotherapy treatment for cancer. While the bone marrow is functioning below normal levels, the patient is at risk, and needs to be monitored very closely. In some cases, hospitalization is recommended for people with Bone marrow suppression until their bone marrow is functioning normally. In recent years, some TCMs have been reported to have beneficial effects on chemotherapyrelated bone marrow suppression. Colla corii asini (or E-Jiao in Chinese), donkey-hide gelatin prepared by stewing and concentrating from Equus asinus L. donkey hide, is a health-care food and traditional Chinese medicine widely used in life-nourishing and clinical hematic antanemic therapy for more than 2,000 years in China (32). Many studies indicated that E-Jiao and its preparations such as Fufang E-Jiao Jiang could effectively promote the recovery of bone marrow hemopoietic function in cancer patients with myelosuppression who were undergoing chemotherapy. Fufang E-Jiao Jiang in combination with conventional interleukin-11 (rhIL-11) and recombinant human granulocyte colony stimulating factor (rhG-CSF) in cancer patients showed significant effects on relieving the myelosuppression caused by GP (Gemcitabine + DDP) regimen chemotherapy and increasing white cell and blood platelet counts compared to using rhIL-11 and rhG-CSF groups alone (96). In addition, TJ-48 was reported to have significant effects on alleviating myelosuppression caused by FOLFOX4 regimen chemotherapy in patients with gastrointestinal malignant tumor. It could effectively increase white cell counts and improve symptoms such as dizziness and fatigue (97). In all, TCM interventions appear to have beneficial effects on alleviating myelosuppression caused by chemotherapy in cancer patients. However, there are several limitations associated with the current published studies on TCMs relieving myelosuppression such as indeterminate results, small sample sizes, and little examination of outcomes. Thus, conducting rigorously designed trials on potential Chinese herbal medicine are warranted.

\subsection{Radiation pneumonitis}

Radiation pneumonitis is one of the most common complications during radiotherapy of thoracic tumors. It impacts the quality of life of the patients and has a lifethreatening danger. However, there is a lack of drugs for prevention and treatment of this disease. In recent years, some TCMs have been reported to have beneficial effects on radiotherapy-related radiation pneumonitis. 
Shenqi fuzheng injection combined with antibiotics and short-term pulse therapy with highly efficient hormones had a good effect for radiation pneumonitis compared to the control group treated only by antibiotic and hormone pulse therapy. The combination could effectively improve respiratory symptoms and signs, and regulate subsets of $\mathrm{T}-1 \mathrm{ymphocytes}(\mathrm{CD} 3+$, CD4+, CD8+ and CD4+/CD8+ ratio), as well as increase patients' quality of life (98). In addition, a prospective randomized clinical study was conducted to assess the effect of a Traditional Chinese herbal formulation composed of Liangxue Jiedu Huoxue Decoction including 7 herbs (Radix rehmanniae, Rhizoma chuanxiong, Cortex moutan, Peach seed, Flos carthami, Radix astragali, and Fructus forsythiae). In this study, 100 lung cancer patients scheduled to receive radiotherapy were randomly divided into a treatment group (Liangxue Jiedu Huoxue Decoction+ radiotherapy) and control group (radiotherapy) with 50 patients in each group (99). Results showed that the incidence rate of radiation pneumonitis was lower in the treatment group than in the control group (13.04\% versus $33.33 \%, p<0.05)$. Furthermore, the extent of lung injures and the symptoms of radiation pneumonitis improved in the treatment group. In all, TCM interventions appear to have beneficial effects on alleviating radiation pneumonitis caused by radiotherapy in cancer patients. However, there are several limitations associated with the current published studies on TCMs relieving radiation pneumonitis such as indeterminate results, small sample sizes, and little examination of outcomes. Thus, conducting rigorously designed trials on potential Chinese herbal medicine are warranted.

\subsection{Hepatotoxicity}

Hepatotoxicity is a common side effect of chemotherapy. Its prevalence ranges from 33 to $65.6 \%$ among patients with cancer, and up to $30 \%$ of patients have grade III or IV hepatotoxicity. Although $90 \%$ of patients resumed normal liver function after stopping use of hepatotoxic drugs, current chemotherapeutic guidelines require stopping chemotherapy when hepatotoxicity as indicated by AST and ALT levels, reaches four times the upper normal limit. Moreover, there is a lack of drugs that effectively protect liver function and elevation of liver enzymes (12). In recent years, some TCMs have been reported to have beneficial effects on improving hepatotoxicity. To examine the effectiveness of TCM for protecting the liver from the toxic effects of chemotherapy among patients with cancer receiving joint TCM (e.g., Xiao-chai-hu-tang, Huang-lian-jiedu-tang or Yin-chen-wu-ling-san) and chemotherapy, a case-control study involving 89 patients and 184 chemotherapy courses was conducted (100). Use of TCM with chemotherapy resulted in protection of the liver during chemotherapy, as manifested by lower serum AST and ALT levels. However, this study only examined a limited spectrum of patient outcomes. A future study with more-homogeneous samples and a larger sample size is needed.

\section{Conclusion}

In conclusion, TCM as adjuvant cancer treatment plays important roles in different stages of cancer lesions including post-operation, radiotherapy or chemotherapy stages. Some TCMs (e.g., TJ-41, Liujun-zi-tang, PHY906, Coumarin, and Aescine) are capable of improving the post-operation symptoms such as fatigue, pain, appetite, diarrhea, nausea, vomiting, and lymphedema. Some TCMs (e.g., Ginseng, Huang-Qi, BanZhiLian, TJ-48, Huachansu injection, Shenqi fuzheng injection, and Kanglaite injection) in combination with chemo- or radiotherapy are capable of enhancing the efficacy of and diminishing the side effects and complications caused by chemo- and radiotherapy. Taken together, they have great advantages in terms of suppressing tumor progression, relieving surgery complications, increasing the sensitivity of chemo- and radiotherapeutics, improving an organism's immune system function, and lessening the damage caused by surgery, chemo- or radio-therapeutics. They have significant effects on relieving breast cancer-related lymphedema, reducing cancer-related fatigue and pain, improving radiation pneumonitis and gastrointestinal side effects, protecting liver function, and even ameliorating bone marrow suppression. This review of those medicines should contribute to an understanding of Chinese herbal medicines as an adjunctive therapy in the whole course of cancer treatment instead of only the terminal stage of cancer, with providing useful information for development of more effective anti-cancer drugs and making more patients "survival with cancer" for a long time. However, rigorously designed trials on potential Chinese herbal medicine must be further examined involving cancer patients in the future.

\section{References}

1. WHO (2012). Non-Communicable Diseases. Burden: Mortality, Morbidity and Risk Factors. http://www.who. int/nmh/publications/ncd_report_chapter1.pdf (Accessed October, 24, 2014).

2. Hao J, Chen WQ. 2012 Chinese Cancer Registry Annual Report. National Cancer Center, Disease prevention and control bureau, Ministry of Health.

3. Targeted therapy. http://en.wikipedia.org/wiki/Targeted_ therapy (Accessed October, 24, 2014).

4. Mellman I, Coukos G, Dranoff G. Cancer immunotherapy comes of age. Nature. 2011; 480:480-489.

5. Chen M, Guo Y. Consideration about the concept of "tumor-bearing survival". Zhong Hua Zhong Yi Yao Za 
Zhi. 2012; 27:3193-3194. (in Chinese)

6. Li X, Yang G, Li X, Zhang Y, Yang J, Chang J, Sun X, Zhou X, Guo Y, Xu Y, Liu J, Bensoussan A. Traditional Chinese medicine in cancer care: A review of controlled clinical studies published in Chinese. PLoS One. 2013; 8:e60338.

7. Zhou J, Zhou T, Jiang M, Wang X, Liu Q, Zhan Z, Zhang X. Research progress on synergistic anti-tumor mechanisms of compounds in traditional Chinese medicine. J Tradit Chin Med. 2014; 34:100-105.

8. Liang W, Yew DT, Hon KL, Wong CK, Kwok TC, Leung PC. Indispensable value of clinical trials in the modernization of traditional Chinese medicine: 12 years' experience at CUHK and future perspectives. Am J Chin Med. 2014; 42:587-604.

9. Ling CQ. My reviews on the etiology and pathogenesis of malignant cancer. Journal of Traditional Chinese Medicine. 2009; 50:952-953. (in Chinese)

10. Smith ME, Bauer-Wu S. Traditional Chinese Medicine for cancer-related symptoms. Semin Oncol Nurs. 2012; 28:64-74.

11. Chao TH, Fu PK, Chang CH, Chang SN, Chiahung Mao F, Lin CH; Evidence-based Chinese Medicine Research Group. Prescription patterns of Chinese herbal products for post-surgery colon cancer patients in Taiwan. J Ethnopharmacol. 2014; 155:702-708.

12. Qi F, Li A, Inagaki Y, Gao J, Li J, Kokudo N, Li XK, Tang W. Chinese herbal medicines as adjuvant treatment during chemo- or radio-therapy for cancer. Biosci Trends. 2010; 4:297-307.

13. Ling $C Q$, Yue $X Q$, Ling $C$. Three advantages of using traditional Chinese medicine to prevent and treat tumor. J Integr Med. 2014; 12:331-335.

14. Konkimalla VB, Efferth T. Evidence-based Chinese medicine for cancer therapy. J Ethnopharmacol. 2008; 116:207-210.

15. Xiang YZ, Shang HC, Gao XM, Zhang BL. A comparison of the ancient use of Ginseng in traditional Chinese medicine with modern pharmacological experiments and clinical trials. Phytother Res. 2008; 22:851-858.

16. Lee SY, Kim YK, Park N, Kim CS, Lee CY, Park SU. Chemical constituents and biological activities of the berry of Panax ginseng. J Med Plants Res. 2010; 5:349-353.

17. Sun YX. Structure and biological activities of the polysaccharides from the leaves, roots and fruits of Panax ginseng C.A. Meyer: An overview. Carbohydr Polym. 2011; 85:490-499.

18. Hofseth LJ, Wargovich MJ. Inflammation, cancer, and targets of ginseng. J Nutr. 2007; 137 (Suppl 1):183S-185S.

19. Yun TK. Experimental and epidemiological evidence on nonorgan specific cancer preventive effect of Korean ginseng and identification of active compounds. Mutat Res. 2003; 523-524:63-74.

20. Lee JH, Kwon KR, Cho CK, Han SS, Yoo HS. Advanced cancer cases treated with cultivated wild ginseng phamacopuncture. J Acupunct Meridian Stud. 2010; 3:119-124.

21. Barton DL, Liu H, Dakhil SR, Linquist B, Sloan JA, Nichols CR, McGinn TW, Stella PJ, Seeger GR, Sood A, Loprinzi CL. Wisconsin Ginseng (Panax quinquefolius) to improve cancer-related fatigue: A randomized, doubleblind trial, N07C2. J Natl Cancer Inst. 2013; 105:12301238.

22. Lee JM, Hah JO, Kim HS. The effect of red ginseng extract on inflammatory cytokines after chemotherapy in children. J Ginseng Res. 2012; 36:383-390.

23. Lee TK, Johnke RM, Allison RR, O'Brien KF, Dobbs LJ Jr. Radioprotective potential of ginseng. Mutagenesis. 2005; 20:237-243.

24. Qi LW, Cao J, Li P, Yu QT, Wen XD, Wang YX, Li CY, Bao KD, Ge XX, Cheng XL. Qualitative and quantitative analysis of Radix Astragali products by fast highperformance liquid chromatography-diode array detection coupled with time-of-flight mass spectrometry through dynamic adjustment of fragmentor voltage. J Chromatogr A. 2008; 1203:27-35.

25. Yan MM, Liu W, Fu YJ, Zu YG, Chen CY, Luo M. Optimisation of the microwave-assisted extraction process for four main astragalosides in Radix Astragali. Food Chem. 119:1663-1670.

26. Li J, Bao Y, Lam W, Li W, Lu F, Zhu X, Liu J, Wang H. Immunoregulatory and anti-tumor effects of polysaccharopeptide and Astragalus polysaccharides on tumor-bearing mice. Immunopharmacol Immunotoxicol. 2008; 30:771-782.

27. Law PC, Auyeung KK, Chan LY, Ko JK. Astragalus saponins downregulate vascular endothelial growth factor under cobalt chloride-stimulated hypoxia in colon cancer cells. BMC Complement Altern Med. 2012; 12:160.

28. Lin YW, Chiang BH. Anti-tumor activity of the fermentation broth of Cordyceps militaris cultured in the medium of Radix astragali. Process Biochem. 2008; 43:244-250.

29. Taixiang W, Munro AJ, Guanjian L. Chinese medical herbs for chemotherapy side effects in colorectal cancer patients. Cochrane Database Syst Rev. 2005; 1:CD004540.

30. Yan PH, Yan M, Wang XM, Wang SH. Effect of Huangqi injection on short-term prognosis in children with acute lymphoblastic leukemia. Zhongguo Dang Dai Er Ke Za Zhi. 2014; 16:141-146. (in Chinese)

31. Li Y, Cui HJ, Huang JC, Wu XQ. Clinical study of Jiawei Huangqi Guizhi Wuwu Decoction in preventing and treating peripheral neuro-sensory toxicity caused by oxaliplatin. Chin J Integr Med. 2006; 12:19-23.

32. The State Pharmacopoeia Commission of the People's Republic of China. Pharmacopoeia of the People's Republic of China 2010. China Chemical Industry Press, Beijing, China, 2010.

33. Yang C, Gou Y, Chen J, An J, Chen W, Hu F. Structural characterization and antitumor activity of a pectic polysaccharide from Codonopsis pilosula. Carbohydr Polym. 2013; 98:886-895.

34. Xin T, Zhang F, Jiang Q, Chen C, Huang D, Li Y, Shen W, Jin Y, Sui G. The inhibitory effect of a polysaccharide from Codonopsis pilosula on tumor growth and metastasis in vitro. Int J Biol Macromol. 2012; 51:788-793.

35. Sun Y. Biological activities and potential health benefits of polysaccharides from Poria cocos and their derivatives. Int J Biol Macromol. 2014; 68:131-134.

36. Ríos JL. Chemical constituents and pharmacological properties of Poria cocos. Planta Med. 2011; 77:681-691.

37. Cheng S, Eliaz I, Lin J, Thyagarajan-Sahu A, Sliva D. Triterpenes from Poria cocos suppress growth and invasiveness of pancreatic cancer cells through the downregulation of MMP-7. Int J Oncol. 2013; 42:18691874.

38. Kikuchi T, Uchiyama E, Ukiya M, Tabata K, Kimura Y, Suzuki T, Akihisa T. Cytotoxic and apoptosis-inducing activities of triterpene acids from Poria cocos. J Nat Prod. 2011; 74:137-144. 
39. Chen M, May BH, Zhou IW, Xue CC, Zhang AL. FOLFOX 4 combined with herbal medicine for advanced colorectal cancer: A systematic review. Phytother Res. 2014; 28:976-991.

40. Niu Y, Meng QX. Chemical and preclinical studies on Hedyotis diffusa with anticancer potential. J Asian Nat Prod Res. 2013; 15:550-565.

41. Kuo YJ, Yang JS, Lu CC, Chiang SY, Lin JG, Chung JG. Ethanol extract of Hedyotis diffusa willd upregulates G0/G1 phase arrest and induces apoptosis in human leukemia cells by modulating caspase cascade signaling and altering associated genes expression was assayed by cDNA microarray. Environ Toxicol. 2014; doi:10.1002/ tox.21989.

42. Cai Q, Lin J, Wei L, Zhang L, Wang L, Zhan Y, Zeng J, Xu W, Shen A, Hong Z, Peng J. Hedyotis diffusa Willd inhibits colorectal cancer growth in vivo via inhibition of STAT3 signaling pathway. Int J Mol Sci. 2012; 13:61176128.

43. Lee SZ, Huang TC, Wei MC, Yang YC. Extraction of protocatechuic acid from Scutellaria barbata D. Don using supercritical carbon dioxide. J Supercrit Fluid. 2013; 81:55-66.

44. Nie XP, Qu GW, Yue XD, Li GS, Dai SJ. Scutelinquanines A-C, three new cytotoxic neo-clerodane diterpenoid from Scutellaria barbata. Phytochem Lett. 2010; 3:190-193.

45. Lin J, Chen Y, Cai Q, Wei L, Zhan Y, Shen A, Sferra TJ, Peng J. Scutellaria Barbata D Don Inhibits Colorectal Cancer Growth via Suppression of Multiple Signaling Pathways. Integr Cancer Ther. 2013; 13:240-248.

46. Perez AT, Arun B, Tripathy D, Tagliaferri MA, Shaw HS, Kimmick GG, Cohen I, Shtivelman E, Caygill KA, Grady D, Schactman M, Shapiro CL. A phase 1B dose escalation trial of Scutellaria barbata (BZL101) for patients with metastatic breast cancer. Breast Cancer Res Treat. 2010; 120:111-118.

47. Chen XP, Li W, Xiao XF, Zhang LL, Liu CX. Phytochemical and pharmacological studies on Radix Angelica sinensis. Chin J Nat Med. 2013; 11:577-587.

48. Chao WW, Lin BF. Bioactivities of major constituents isolated from Angelica sinensis (Danggui). Chinese Medicine. 2011; 6:29.

49. Kou W, Li YD, Liu K, Sun SB, Dong YM, Wu ZH. Radix Angelicae Sinensis and Radix Hedysari enhance radiosensitivity of $12 \mathrm{C} 6+$ radiation in human liver cancer cells by modulating apoptosis protein. Saudi Med J. 2014; 35:945-952.

50. Kumar P, Kadakol A, Shasthrula P, Mundhe NA, Jamdade VS, Barua CC, Gaikwad AB. Curcumin as an Adjuvant to Breast Cancer Treatment. Anticancer Agents Med Chem. 2015. [Epub ahead of print]

51. Goel A, Aggarwal BB. Curcumin, the golden spice from Indian saffron, is a chemosensitizer and radiosensitizer for tumors and chemoprotector and radioprotector for normal organs. Nutr Cancer. 2010; 62:919-930.

52. Qi FH, Wang ZX, Cai PP, Zhao L, Gao JJ, Kokudo N, Li AY, Han JQ, Tang W. Traditional Chinese medicine and related active compounds: A review of their role on hepatitis B virus infection. Drug Discov Ther. 2013; 7:212-224.

53. Kido T, Mori K, Daikuhara H, Tsuchiya H, Ishige A, Sasaki H. The protective effect of hochu-ekki-to (TJ41), a Japanese herbal medicine, against HSV-1 infection in mitomycin C-treated mice. Anticancer Res. 2000; 20:4109-4113.
54. Kimura M, Sasada T, Kanai M, Kawai Y, Yoshida Y, Hayashi E, Iwata S, Takabayashi A. Preventive effect of a traditional herbal medicine, Hochu-ekki-to, on immunosuppression induced by surgical stress. Surg Today. 2008; 38:316-322.

55. Jeong JS, Ryu BH, Kim JS, Park JW, Choi WC, Yoon SW. Bojungikki-tang for cancer-related fatigue: A pilot randomized clinical trial. Integr Cancer Ther. 2010; 9:331338.

56. Ogawa K, Omatsu T, Matsumoto C, Tsuchiya N, Yamamoto M, Naito Y, Yoshikawa T. Protective effect of the Japanese traditional medicine juzentaihoto on myelosuppression induced by the anticancer drug TS-1 and identification of a potential biomarker of this effect. BMC Complement Altern Med. 2012; 12:118.

57. Huang SM, Chien LY, Tai CJ, Chiou JF, Chen CS, Tai CJ. Effectiveness of 3-week intervention of Shi Quan $\mathrm{Da} \mathrm{Bu}$ Tang for alleviating hematotoxicity among patients with breast carcinoma receiving chemotherapy. Integr Cancer Ther. 2013; 12:136-144.

58. Tsuchiya M, Kono H, Matsuda M, Fujii H, Rusyn I. Protective effect of Juzen-taiho-to on hepatocarcinogenesis is mediated through the inhibition of Kupffer cell-induced oxidative stress. Int J Cancer. 2008; 123:2503-2511.

59. Ikemoto T, Shimada M, Iwahashi S, Saito Y, Kanamoto M, Mori H, Morine Y, Imura S, Utsunomiya T. Changes of immunological parameters with administration of Japanese Kampo medicine (Juzen-Taihoto/TJ-48) in patients with advanced pancreatic cancer. Int J Clin Oncol. 2014; 19:81-86.

60. Liu SH, Cheng YC. Old formula, new Rx: The journey of PHY906 as cancer adjuvant therapy. J Ethnopharmacol. 2012; 140:614-623.

61. Rockwell S, Grove TA, Liu Y, Cheng YC, Higgins SA, Booth CJ. Preclinical studies of the Chinese herbal medicine formulation PHY906 (KD018) as a potential adjunct to radiation therapy. Int J Radiat Biol. 2013; 89:16-25.

62. Saif MW, Li J, Lamb L, Kaley K, Elligers K, Jiang Z, Bussom S, Liu SH, Cheng YC. First-in-human phase II trial of the botanical formulation PHY 906 with capecitabine as second-line therapy in patients with advanced pancreatic cancer. Cancer Chemother Pharmacol. 2014; 73:373-380.

63. Qi FH, Wang ZX, Cai PP, Zhao L, Gao JJ, Kokudo N, Li AY, Han JQ, Tang W. Traditional Chinese medicine and related active compounds: A review of their role on hepatitis B virus infection. Drug Discov Ther. 2013; 7:212-224

64. Zheng CS, Wu YS, Bao HJ, Xu XJ, Chen XQ, Ye HZ, Wu GW, Xu HF, Li XH, Chen JS, Liu XX. Understanding the polypharmacological anticancer effects of Xiao Chai $\mathrm{Hu}$ Tang via a computational pharmacological model. Exp Ther Med. 2014; 7:1777-1783.

65. Li J, Xie M, Gan Y. Effect of Xiaochaihu decoction and different herbal formulation of component on inhibiting H22 liver cancer in mice and enhancing immune function. Zhongguo Zhong Yao Za Zhi. 2008; 33:1039-1044. (in Chinese)

66. Rino Y, Tarao K, Morinaga S, Ohkawa S, Miyakawa K, Hirokawa S, Masaki T, Tarao N, Yukawa N, Saeki H, Takanashi Y, Imada T. Reduction therapy of alanine aminotransferase levels prevent HCC development in patients with $\mathrm{HCV}$-associated cirrhosis. Anticancer Res. 2006; 26:2221-2226. 
67. Matsuoka H, Mizushima Y, Kawano M, Tachibana N, Sawada Y, Kato S, Nagakura H, Tanaka M, Suzuki K, Tadanobu K. Clinical availability of the herbal medicine, SYOUSAIKOTOU, as a gargling agent for prevention and treatment of chemotherapy-induced stomatitis. Gan To Kagaku Ryoho. 2004; 31:2017-2020. (in Japanese)

68. Wang S, Wu X, Tan M, Gong J, Tan W, Bian B, Chen M, Wang Y. Fighting fire with fire: Poisonous Chinese herbal medicine for cancer therapy. J Ethnopharmacol. 2012; 140:33-45.

69. Li J, Wang JC, Ma B, Gao W, Chen P, Sun R, Yang KH. Shenqi Fuzheng Injection (SFI) for advanced gastric cancer: A systematic review of randomized controlled trials. Chin J Integr Med. 2015; 21:71-79.

70. Dong J, Su SY, Wang MY, Zhan Z. Shenqi fuzheng, an injection concocted from Chinese medicinal herbs, combined with platinum-based chemotherapy for advanced non-small cell lung cancer: A systematic review. J Exp Clin Cancer Res. 2010; 29:137.

71. Guo ML, Chen HY, Han XL, Zhang HZ. Influence of Shenqi fuzheng injection combined with radiochemotherapy on quality of life in head and neck cancer patients. Modern Journal of Integrated Traditional Chinese and Western Medicine. 2014; 23:1273-1275. (in Chinese)

72. Liu X, Yang Q, Xi Y, Yu K, Wang W, Zhao X, Kou X. Kanglaite injection combined with chemotherapy versus chemotherapy alone in the treatment of advanced nonsmall cell lung carcinoma. J Cancer Res Ther. 2014; 10 (Suppl 1):46-51.

73. Fu F, Wan Y, Mulati, Wu T. Kanglaite injection combined with hepatic arterial intervention for unresectable hepatocellular carcinoma: A meta-analysis. J Cancer Res Ther. 2014; 10 (Suppl 1):38-41.

74. Wang JC, Tian JH, Ge L, Gan YH, Yang KH. Which is the best Chinese herb injection based on the FOLFOX regimen for gastric cancer? A network meta-analysis of randomized controlled trials. Asian Pac J Cancer Prev. 2014; 15:4795-4800.

75. Qi F, Li A, Inagaki Y, Kokudo N, Tamura S, Nakata M, Tang W. Antitumor activity of extracts and compounds from the skin of the toad Bufo bufo gargarizans Cantor. Int Immunopharmacol. 2011; 11:342-349.

76. Qi F, Li A, Inagaki Y, Xu H, Wang D, Cui X, Zhang L, Kokudo N, Du G, Tang W. Induction of apoptosis by cinobufacini preparation through mitochondria- and Fas-mediated caspase-dependent pathway. Food Chem Toxicol. 2012; 50:295-302.

77. Meng Z, Yang P, Shen Y, Bei W, Zhang Y, Ge Y, Newman RA, Cohen L, Liu L, Thornton B, Chang DZ, Liao Z, Kurzrock R. Pilot study of huachansu in patients with hepatocellular carcinoma, nonsmall-cell lung cancer, or pancreatic cancer. Cancer. 2009; 115:5309-5318.

78. Qin TJ, Zhao XH, Yun J, Zhang LX, Ruan ZP, Pan BR. Efficacy and safety of gemcitabine-oxaliplatin combined with huachansu in patients with advanced gallbladder carcinoma. World J Gastroenterol. 2008; 14:5210-5216.

79. Chen Z, Chen HY, Lang QB, Li B, Zhai XF, Guo YY, Yue XQ, Ling CQ. Preventive effects of jiedu granules combined with cinobufacini injection versus transcatheter arterial chemoembolization in post-surgical patients with hepatocellular carcinoma: A case-control trial. Chin J Integr Med. 2012; 18:339-344.

80. Sun T, Zhang Y, Shen Y, Hu K, Zuo M. A case of advanced lung cancer with malignant pericardial effusion treated by intrapericardial Cinobufacini injection instillation. Biosci
Trends. 2014; 8:235-239.

81. Wang MJ, Nan X, Feng G, Yu HT, Hu GF, Liu YQ. Design, synthesis and bioactivity evaluation of novel acylthiourea derivatives of cantharidin. Ind Crop Prod. 2014; 55:11-18.

82. Han L, Sun YJ, Pan YF, Ding H, Chen X, Zhang X. Cantharidin combined with chemotherapy for Chinese patients with metastatic colorectal cancer. Asian Pac J Cancer Prev. 2014; 15:10977-10979.

83. Ji ZQ, Huang XE, Wu XY, Liu J, Wang L, Tang JH. Safety of Brucea javanica and cantharidin combined with chemotherapy for treatment of NSCLC patients. Asian Pac J Cancer Prev. 2014; 15:8603-8605.

84. Patricolo GE, Armstrong K, Riutta J, Lanni T. Lymphedema care for the breast cancer patient: An integrative approach. Breast. 2015; 24:82-85.

85. Wanchai A, Armer JM, Stewart BR. Complementary and alternative medicine and lymphedema. Semin Oncol Nurs. 2013; 29:41-49.

86. Casley-Smith JR, Morgan RG, Piller NB. Treatment of lymphedema of the arms and legs with 5,6-benzo-[alpha]pyrone. N Engl J Med. 1993; 329:1158-1163.

87. Loprinzi CL, Kugler JW, Sloan JA, Rooke TW, Quella SK, Novotny P, Mowat RB, Michalak JC, Stella PJ, Levitt $\mathrm{R}$, Tschetter LK, Windschitl H. Lack of effect of coumarin in women with lymphedema after treatment for breast cancer. N Engl J Med. 1999; 340:346-350.

88. Jiang ZM. Upper extremity lymphedema treated with compound Diosmin and Sodium aescine after breast cancer surgery. World Journal of Integrated Traditional and Western Medicine. 2010; 5:341-342. (in Chinese)

89. Takeda H, Muto S, Nakagawa K, Ohnishi S, Sadakane C, Saegusa Y, Nahata M, Hattori T, Asaka M. Rikkunshito as a ghrelin enhancer. Methods Enzymol. 2012; 514:333351.

90. Gunji S, Ueda S, Yoshida M, Kanai M, Terajima H, Takabayashi A. Effects of rikkunshito, a kampo medicine, on quality of life after proximal gastrectomy. J Surg Res. 2013; 185:575-580.

91. Chen HY, Li SG, Cho WC, Zhang ZJ. The role of acupoint stimulation as an adjunct therapy for lung cancer: A systematic review and meta-analysis. BMC Complement Altern Med. 2013; 13:362.

92. Wang SJ, Xu J, Gong DD, Man CF, Fan Y. Meta-analysis of oral Chinese herbal medicine as an adjuvant treatment in relieving pain secondary to bone metastases. Chin J Integr Med. 2013 Oct 14. [Epub ahead of print]

93. Yanju B, Yang L, Hua B, Hou W, Shi Z, Li W, Li C, Chen C, Liu R, Qin Y, Lv W. A systematic review and meta-analysis on the use of traditional Chinese medicine compound kushen injection for bone cancer pain. Support Care Cancer. 2014; 22:825-836.

94. Su CX, Wang LQ, Grant SJ, Liu JP. Chinese herbal medicine for cancer-related fatigue: A systematic review of randomized clinical trials. Complement Ther Med. 2014; 22:567-579.

95. Lo LC, Chen CY, Chen ST, Chen HC, Lee TC, Chang CS. Therapeutic efficacy of traditional Chinese medicine, Shen-Mai San, in cancer patients undergoing chemotherapy or radiotherapy: Study protocol for a randomized, double-blind, placebo-controlled trial. Trials. 2012; 13:232.

96. Fu L, Fu H, Liu LQ, Huo ZJ, Yu YH, Yu JM. Efficacy of donkey-hide gelatin mixture for gemcitabine and cisplatin chemotherapy regimen induced myelosuppression. 
Chinese Clinical Oncology. 2014; 19:739-742. (in Chinese)

97. Zhang PY, Qiu N. The clinical observation on Shiquan-da-bu-tang relieving myelosuppression caused by chemotherapy. Modern Journal of Integrated Traditional Chinese and Western Medicine. 2009; 18:2157-2158. (in Chinese)

98. Zheng W, Gao ZH, Wu LN. Clinical observation on treatment of radiative pneumonia in patients with lung cancer by integrative Chinese and Western medicine. Zhongguo Zhong Xi Yi Jie He Za Zhi. 2007; 27:11211123. (in Chinese)
99. Xiao C, Ding HJ, Feng LC, Qu BL, Dou YQ. Efficacy of Liangxue Jiedu Huoxue Decoction in prevention of radiation pneumonitis: A randomized controlled trial. Zhong Xi Yi Jie He Xue Bao. 2010; 8:624-628. (in Chinese)

100. Liu ML, Chien LY, Tai CJ, Lin KC, Tai CJ. Effectiveness of Traditional Chinese Medicine for Liver Protection and Chemotherapy Completion among Cancer Patients. Evid Based Complement Alternat Med. 2011; 2011:291843.

(Received January 10, 2015; Revised February 20, 2015; Accepted February 23, 2015) 